\title{
Assessing the impact of clinical pharmacy services on the healthcare outcomes of patients attending an outpatient haemodialysis unit in a rural hospital in Egypt: a quasi-experimental study
}

\author{
Reem Nagib',(1), Maha Abdul-Latif2,(1), Hoda S. Sakoury',(1), \\ Mahmoud E. Elrggal ${ }^{3, *},\left(\right.$ ) , Ejaz Cheema ${ }^{4,(1)}$, Mohamed Hassan Elnaem ${ }^{5,6,(1)}$ \\ and Karim A. El-Fass ${ }^{7}$, (i)
}

'Ministry of Health, Alexandria, Egypt

${ }^{2}$ Appalachian College of Pharmacy, Oakwood, VA, USA

${ }^{3}$ Clinical Pharmacy Department, Umm Al Qura University, Mecca, Saudi Arabia

${ }^{4}$ School of Pharmacy, University of Birmingham, Edgbaston, Birmingham, UK

${ }^{5}$ Department of Pharmacy Practice, Faculty of Pharmacy, International Islamic University Malaysia, Selangor, Malaysia

${ }^{6}$ Quality Use of Medicines Research Group, Faculty of Pharmacy, International Islamic University Malaysia, Selangor, Malaysia

'Pharmacy Practice Department, College of Clinical Pharmacy, King Faisal University, Hofuf, Al-Ahsa, Kingdom of Saudi Arabia

*Correspondence: Karim A. El-Fass, College of Clinical Pharmacy, King Faisal University, Hofuf, Al-Ahsa, Kingdom of Saudi Arabia. Emails: kelfass@kfu.edu.sa, kareem.elfass@gmail.com

Received October 18, 2020; Accepted June 15, 2021.

\begin{abstract}
Objectives This study aimed to investigate the impact of newly introduced clinical pharmacy services on the health care of chronic haemodialysis patients attending an outpatient haemodialysis unit in a rural hospital with limited resources in Alexandria, Egypt.

Methods A quasi-experimental pre-/post-test study was conducted from November 2016 till June 2018. Clinical pharmacists collected relevant information using a pre-specified form. Patients' data were reviewed for drug-related problems (DRPs), which were documented using the Pharmaceutical Care Network Europe (PCNE) classification system, version 8.02 and resolved by the clinical pharmacists. Measured values of calcium, phosphorus and haemoglobin were compared with target levels set by the Kidney Disease: Improving Global Outcomes (KDIGO) guidelines. Proportions of patients achieving therapeutic values of each parameter were compared before and after implementing the program using the McNemar test. We also reported the analysis of DRPs identified and addressed by the clinical pharmacy team.

Key findings A total of 685 DRPs were identified during the follow-up period. Improper dose and inappropriate drug selection were the most common DRPs $(45.8 \%$ and $18.9 \%$, respectively). There was a statistically significant increase in the proportion of patients achieving target corrected calcium levels (30\% pre-intervention versus $69.6 \%$ post-intervention, $P=0.001)$ and haemoglobin
\end{abstract}


levels $(9.1 \%$ pre-intervention versus $31.9 \%$ post-intervention, $P=0.003)$, but not in phosphorus levels (18.6\% pre-intervention versus $22 \%$ post-intervention, $P=0.7$ ).

Conclusions Clinical pharmacists were able to resolve frequent DRPs and improve some markers of health care in haemodialysis patients.

Keywords: clinical pharmacy; corrected calcium; haemoglobin; drug-related problems; haemodialysis

\section{Introduction}

Haemodialysis patients are at an increased risk of developing comorbidities and complications due to their advanced renal disease and associated renal replacement therapy. Such a complicated health state often requires multiple medications which can subsequently impact how patients comply with their prescribed medication regimens and predispose them to drug-related problems (DRPs). ${ }^{[1,2]}$ Furthermore, psychological and socioeconomic factors impose an additional burden on haemodialysis patients, their care providers and healthcare systems. However, evidence suggests that improvements brought about by advances in dialysis modalities and technologies, as well as manoeuvres for better medical management can help improve the clinical outcomes of haemodialysis patients. ${ }^{[3-5]}$ For example, rigorous compliance with the guidelines for managing common conditions like anaemia, mineral bone disease $(\mathrm{MBD})$ and hypertension was associated with a reduction in all-cause mortality among haemodialysis patients in a large cohort of European dialysis patients (EURODOPPS). ${ }^{[4]}$ Evidence also suggests that certain pharmacological interventions may improve cardiovascular outcomes in haemodialysis patients..$^{[5]}$

In addition to the pharmacological interventions, advances in healthcare systems have also been associated with improved processes and outcomes in haemodialysis patients. For example, the adoption of an integrated care model provided by the Medicare Advantage program was associated with $9 \%$ reduction in mortality when compared with usual care. ${ }^{[6]}$ Similarly, the adoption of a multidisciplinary team with the integration of allied healthcare providers including pharmacists, dietitians and social workers has led to improved care for haemodialysis patients. ${ }^{[7-9]}$ Pharmacists, as members of the multidisciplinary team, can conduct medication reconciliation, reduce medication errors and improve patients' adherence to the usually complex medication regimens in haemodialysis patients. ${ }^{[10-13]}$ Evidence suggests that pharmacist-led medication review was associated with better identification and resolution of DRPs. ${ }^{[14-16]}$ Furthermore, the provision of clinical pharmacy services to haemodialysis patients led to improvement in healthcare outcomes and better management of anaemia and MBD. ${ }^{[17-19]}$

Although clinical pharmacy services have been widely provided to haemodialysis patients in many countries including the USA, some European and some Middle Eastern countries, such services have only been introduced recently in many other countries including Egypt. ${ }^{[20-22]}$ Furthermore, financial constraints coupled with lack of basic healthcare services including access to essential drugs and investigations pose a significant challenge in the implementation of effective services to patients and may interfere with the quality of care provided to haemodialysis patients. To the authors' knowledge, no studies have been conducted to assess the impact of clinical pharmacy services on adult haemodialysis patients in Egypt. This study, therefore, aimed to investigate the impact of introducing a clinical pharmacy services program (CPSP) on selected healthcare outcomes of patients attending a haemodialysis unit in a rural hospital in Alexandria, Egypt.

\section{Methods}

\section{Study design, population and setting}

A quasi-experimental pre-/post-test study was conducted in a haemodialysis unit of a rural hospital in Alexandria, Egypt. All patients above the age of 18 attending the haemodialysis unit from August 2016 till June 2018 were included. The CPSP implementation began in November 2016 and continued till June 2018. Figure 1 illustrates the timeline of the study.

\section{Ethical approval}

Ethical approval was obtained from the Ministry of Health Research Ethics Committee (17-2018/9).

\section{Data collection}

A data collection form was developed, using previous literature, ${ }^{[23]}$ and tailored to provide optimal utility for both clinical and research purposes. The form was used to collect relevant patients' information from medical records and patient interviews conducted by the clinical pharmacists. The data included patient demographics, medical history, medication history, dietary habits, laboratory data, vital signs, information about dialysis sessions, medicines adherence and lifestyle modifications.

\section{Study intervention}

During the program implementation period, pharmacists provided clinical pharmacy services, including identification and resolution of DRPs, patient education about renal disease and haemodialysis, lifestyle modifications and basic self-monitoring skills. DRPs identified and resolved by the clinical pharmacists during the study period were documented and classified using the Pharmaceutical Care Network Europe (PCNE) system, version 8.02. ${ }^{[24]}$ Each problem was described according to four major domains: problem type, cause, intervention type and intervention acceptance.

\section{Outcome measures}

Calcium and phosphorus blood levels were used as indicators for MBD control. Meanwhile, haemoglobin concentration was used

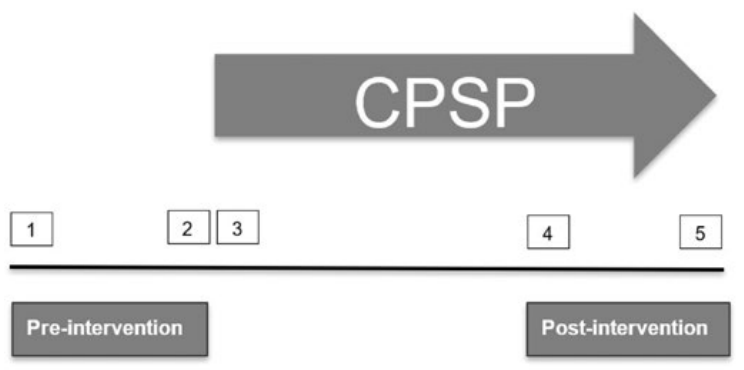

Figure 1 Timeline of the study. 1, August 2016; 2, October 2016; 3, November 2016; 4, April 2018; 5, June 2018. CPSP, Clinical Pharmacy Services Program. 
as the indicator for anaemia control. Measured values were compared with target levels set by the Kidney Disease: Improving Global Outcomes (KDIGO) guidelines, which recommended target haemoglobin levels ranging between 10 and $11.5 \mathrm{~g} / \mathrm{dL}$, and corrected calcium and phosphorus levels within the normal range of each. ${ }^{[25,26]}$ Due to financial constraints, costly laboratory tests like parathyroid hormone (PTH) levels, iron indices were not always performed at the intervals recommended by the KDIGO guidelines, making the available data not enough to be used as outcome measures. Proportions of patients achieving therapeutic values of each parameter were compared before and after implementing the program. Since the KDIGO guidelines recommend that decisions to initiate or modify medication treatments should be based on trends in laboratory values rather than a single measurement, ${ }^{[25]}$ the pre-intervention values were the average of values collected throughout the 3 -month period that preceded initiating the program and the post-intervention values were estimated by averaging the values of each parameter in the last 3 months of the intervention as shown in Figure 1.

\section{Statistical analysis}

Shapiro-Wilk test was conducted to test for normality of continuous variables. The data were not normally distributed and were, therefore, described as medians and interquartile ranges. Categorical variables were described as numbers and percentages. McNemar test was applied for comparing the proportions of patients achieving target laboratory values pre- and post-program implementation. All statistical analyses were conducted using Microsoft Excel 2013 and SPSS V 21.

\section{Results}

\section{Patients' characteristics}

We followed up a total of 51 patients throughout the study period. The patients' median age was 55 years with a median body mass index (BMI) of $27.3 \mathrm{~kg} / \mathrm{m}^{2}$. Table 1 presents an overview of the patients' demographics and common comorbidities.

\section{Assessment of outcome measures}

Regarding the laboratory markers, the proportion of patients who achieved target corrected calcium levels was significantly higher after implementing the program $(30 \%$ pre-intervention versus $69.6 \%$ post-intervention, $P=0.001$ ), and that was also the case

Table 1 Descriptive characteristics of the study population, median (IOR) or $N(\%)$

\begin{tabular}{lc}
\hline Characteristic & Median $(\mathrm{IQR})$ or $N(\%)$ \\
\hline Number of patients & 51 \\
Male & $28(54.9 \%)$ \\
Female & $23(45.1 \%)$ \\
Age $($ years $)$ & $55(43-60)$ \\
BMI $\left(\mathrm{kg} / \mathrm{m}^{2}\right)$ & $27.3(23-31)$ \\
Dialysis vintage (years) & $5(3-7)$ \\
Comorbidities & \\
Hypertension & $35(68.6 \%)$ \\
Diabetes & $10(19.6 \%)$ \\
HCV & $10(19.6 \%)$ \\
Cardiovascular disease & $4(7.8 \%)$ \\
\hline
\end{tabular}

HCV, hepatitis C virus; IQR, interquartile range. for haemoglobin levels $(9.1 \%$ pre-intervention versus $31.9 \%$ postintervention, $P=0.003$ ). The proportion of patients who achieved target phosphorus levels was higher after the program, however, the increase did not reach statistical significance $(18.6 \%$ pre-intervention versus $22 \%$ post-intervention, $P=0.7)$. Figure 2 demonstrates the proportions of patients whose values were in therapeutic range preand post-CPSP implementation.

\section{DRPs identification, resolution and acceptance}

During the follow-up, a total of 685 DRPs was identified by the clinical pharmacists. When DRPs were classified by type, treatment effectiveness problems were the most common type ( $n=294,42.9 \%$ ), and when they were classified by cause, improper dose selection was the most common cause $(n=314,45.8 \%)$, followed by inappropriate drug selection $(n=130,19 \%)$. Expectedly, since most DRPs were caused by improper dose or drug selection, the majority of the clinical pharmacists' interventions was made at the prescriber's level ( $n=499,72.8 \%$ ). Eighty-five percent of the interventions the clinical pharmacist made to resolve DRPs during the follow-up period were accepted. A detailed classification of the identified DRPs using the PCNE V 8.02 system is provided in Table 2.

\section{Discussion}

We found that the introduction of a CPSP to the haemodialysis unit in a hospital located in a rural area was associated with improvement in the proportion of haemodialysis patients who achieved the target values of laboratory markers that reflect the management of MBD and anaemia ${ }^{[25,26]}$ and was also associated with the resolution of many DRPs in this sensitive population.

$\mathrm{MBD}$ is a common problem in haemodialysis patients that is associated with numerous complications including increased risk of fractures and vascular calcification, which are independently associated with increased morbidity and mortality. Thereby, maintaining target levels of corrected calcium, phosphorus and PTH has been linked to improved survival in haemodialysis patients. ${ }^{[25]}$ Our study showed that the CPSP was associated with significant improvement in the proportion of patients achieving target corrected calcium levels. The interventions provided as part of the CPSP included stoppage of vitamin $\mathrm{D}$ analogues that were not indicated, adjusting the dose of calcium-containing phosphate binders and patient education about diet and bone health. However, the improvement in the

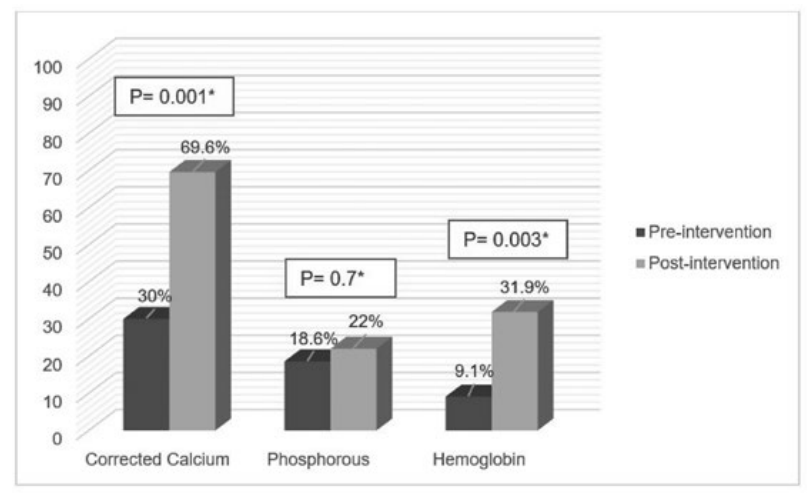

* McNemar test

Figure 2 Proportions of patients whose values were in therapeutic range pre-/post-intervention. 
Table 2 Classification of identified drug-related problems using the PCNE V 8.02 system

\begin{tabular}{|c|c|c|}
\hline Classification of problem (total number of interventions) & Code and description & Frequency and percentage \\
\hline \multirow[t]{3}{*}{ PCNE V8.02 classification of DRPs by problem type (685) } & P1: Treatment effectiveness & $294(42.9)$ \\
\hline & P2: Treatment safety & $193(28.2)$ \\
\hline & P3: Others & $198(28.9)$ \\
\hline \multirow[t]{8}{*}{ PCNE V8.02 classification of DRPs by cause (685) } & C1: Drug selection & $130(18.9)$ \\
\hline & C2: Drug form & $3(0.43)$ \\
\hline & C3: Dose selection & $314(45.83)$ \\
\hline & C4: Treatment duration & $2(0.29)$ \\
\hline & C5: Dispensing & $5(0.73)$ \\
\hline & C6: Drug use/process & $5(0.73)$ \\
\hline & C7: Patient-related & $107(15.9)$ \\
\hline & C8: Other & $119(17.37)$ \\
\hline \multirow[t]{5}{*}{ PCNE V8.02 classification of DRPs by planned intervention (685) } & I0: No intervention & $1(0.147)$ \\
\hline & I1: At prescriber level & $499(72.78)$ \\
\hline & I2: At patient level & $180(26.33)$ \\
\hline & I3: At drug level & 0 \\
\hline & I4: Other & $5(0.74)$ \\
\hline \multirow[t]{3}{*}{ PCNE V8.02 classification of DRPs by intervention acceptance (685) } & A1: Accepted & $583(85)$ \\
\hline & A2: Not accepted & $90(13.2)$ \\
\hline & A3: Other & $12(1.74)$ \\
\hline
\end{tabular}

DRPs, drug-related problems; PCNE V8.02, Pharmaceutical Care Network Europe.

proportion of patients achieving target phosphorus levels was not statistically significant.

Some of our findings are in line with the findings of previous studies. For example, a large retrospective cohort by Roberts-Clary et al. examined the association between the implementation of coordinated pharmacy services and the achievement of target values for MBD management parameters and found that patients who received pharmacy services achieved target calcium, phosphorus, PTH more often than their propensity score-matched controls. ${ }^{[27]}$ On the other hand, a pre-/post-test study conducted by Baker et al. found no significant improvement in MBD parameters to be associated with the implementation of a patient-centred, pharmacist-dietician-led management approach. ${ }^{[28]}$

Anaemia is associated with cardiovascular complications, hospitalization and death in haemodialysis patients. The current guidelines recommend attaining specific values of haemoglobin, ferritin and transferrin saturation (TSAT) to optimize the management of anaemia in dialysis patients. ${ }^{[26]}$ In our study, we included the proportion of patients achieving target haemoglobin levels as a measure for anaemia control, however, we were not able to assess the attainment of target ferritin and TSAT levels as these tests were not performed in our hospital's routine laboratory workup. Nevertheless, clinical pharmacists ensured that each patient had their ferritin and TSAT checked at baseline to determine their eligibility for an iron load. Indeed, most of the patients were severely iron deficient and received loading doses of iron, followed by twice-monthly maintenance doses. Once iron status was corrected, many patients showed remarkable improvement in their haemoglobin levels, which was then followed by adjusting their erythropoietin-stimulating agent (ESA) doses. The CPSP implementation was able to demonstrate a significant improvement in the proportion of patients achieving target haemoglobin levels. However, the overall percentage of patients with controlled haemoglobin levels was not high (around 32\%).

Our results, showing a positive impact of the CPSP on the management of anaemia in haemodialysis patients, came in accordance with the results of other studies that show improved haemoglobin levels. Additionally, some of these studies showed improvements in other anaemia management parameters like TSAT and ferritin. ${ }^{[17-19]}$
As shown in the results, most of the DRPs affected treatment effectiveness and were caused by either suboptimal drug dosing or selection. One example of common DRPs was the presence of significant iron deficiency at baseline in a patient receiving maximal doses of ESA covered by the insurance plan. Subsequently, iron and haemoglobin profiles were significantly improved after receiving an intravenous iron load followed by maintenance doses even in those with insufficient doses of ESA. It is not uncommon that some patients even required a significant ESA dose reduction after that intervention. The preceding example may explain why most interventions were on the prescriber's level, although one of the basic domains of the CPSP was patient education. Furthermore, most of the clinical pharmacist recommendations were accepted (85\%), indicating appropriate collaboration among physicians, pharmacists and patients throughout the program implementation period.

Providing care to haemodialysis patients is usually complex and requires multiple resources, including equipment, investigations, medications and staff. ${ }^{[9]}$ Although evidence suggesting a positive impact of introducing clinical pharmacy services to haemodialysis patients existed, ${ }^{[14,17,26,27,29]}$ it was not clear if these conclusions could be extrapolated to settings with significant financial constraints that may cause shortages in the supply of medications, lack of proper follow-up as well as the low income of the patients rendering them incapable of maintaining their medications. For instance, Ohnishi et al. examined the effect of pharmacist management on haemoglobin levels in haemodialysis patients by developing and applying a hospital-specific protocol for dose adjustments of ESAs and iron products based on haemoglobin, TSAT and ferritin levels. ${ }^{[17]}$ It resulted in a significant increase in haemoglobin levels in the lowhaemoglobin group and a significant decrease in haemoglobin levels in the high-haemoglobin group. While our results come in line with some findings in that study, TSAT and ferritin levels were not always available in our setting. They were only measured at baseline in most patients to determine the patient's eligibility for iron loading and maintenance doses. Moreover, a study conducted in Iran by Dashti et al. encountered some of the barriers we faced in our study, like the unavailability of other phosphate binders than calcium carbonate in the government insurance program. ${ }^{[19]}$ However, 
laboratory parameters were measured regularly, and medications were adjusted accordingly. The results showed an optimization of calcium blood levels and a decrease in phosphorus levels without achieving target levels.

Although clinical pharmacy curricula have been incorporated into pharmacy education and the number of pharmacists holding postgraduate degrees or certificates has been on the rise over the past two decades, the recognition of pharmacists as patient care providers has been relatively slow at practice settings. In 2013, the Egyptian Ministry of Health has declared that establishing clinical pharmacy departments in all hospitals was mandatory and clinical pharmacists had a better chance to assume their roles in patient care. ${ }^{[22,30]}$ To the authors' knowledge, this is the first study that has highlighted the role of clinical pharmacists in managing adult haemodialysis patients in a healthcare setting with limited resources in Egypt. The favourable outcomes reported in this study should encourage wider implementation of clinical pharmacy services in public haemodialysis units.

This study, however, comes with some limitations. It was conducted in a single centre which might prevent the generalization of its results to other units adopting different systems. Moreover, the small sample size might have prevented the detection of a statistically significant difference for phosphorus level improvement. Furthermore, we were not able to follow-up some important laboratory parameters like iron status, PTH and vitamin D levels for all patients on regular bases due to the financial constraints highlighted earlier.

Although patient-reported outcomes - such as patient-reported symptoms - are being advocated for their ability to demonstrate patients' perspectives; and hard clinical outcomes - such as mortality or morbidity end points - are known to provide stronger evidence of the presence or absence of efficacy of interventions, ${ }^{[28,31,32]}$ surrogate parameters like electrolyte and haemoglobin levels were used to derive the end points in this study because they had a well-established clinical utility and their management was within the clinical pharmacists' scope of practice. Moreover, as mentioned in the discussion section, better control of MBD and anaemia is associated with better clinical outcomes such as reduced mortality and hospitalizations, as well as better quality of life for haemodialysis patients. ${ }^{[33]}$ Pharmacoeconomic outcomes were not addressed in the study due to excessive complexity in cost measurement since patients, in some instances, paid for some laboratory investigations and medications. More studies are needed in the future to explore more aspects in which clinical pharmacists can add value to patient care processes and outcomes.

\section{Conclusion}

The introduction of clinical pharmacy services to the haemodialysis unit was associated with significant improvement in the proportion of patients who achieved target levels of corrected calcium and haemoglobin and numerical improvement in the proportion of patients achieving target phosphorus levels. The modest improvement in the achievement of target phosphorus levels denoted the need for further educational efforts about dietary modifications. Moreover, effective phosphate-lowering medications such as non-calciumcontaining phosphate binders were not accessible at the time of the study, because they were not included in the Ministry of Health formulary. Additionally, the relatively low final percentage of patients achieving target haemoglobin levels may be attributed to the inconsistent availability of iron injections as well as the lack of sufficient ESAs doses covered by the patients' insurance program. Moreover, the majority of patients could not afford the high prices of ESAs on their own.

Clinical pharmacists were able to detect and resolve several DRPs that could adversely impact patient outcomes.

These findings support the evidence on the vital role of clinical pharmacists in improving the care provided to haemodialysis patients in settings with economic constraints and should encourage enabling pharmacists to assume wider roles as patient care providers and to incorporate them into decision-making process of establishing institutional and national drug formulary. Future research should be directed to assess more aspects of patient care associated with the introduction of CPSP in healthcare systems with financial challenges.

\section{Acknowledgements}

The authors confirm that this manuscript is original, has not been published elsewhere and is not currently under consideration by another journal, and that all authors have approved the manuscript and agree with its submission to the Journal of Pharmaceutical Health Services Research.

\section{Author Contributions}

R.N., M.A-L., and K.A.E-F. contributed to the design and implementation of the research. H.S.S. and R.N. contributed to data collection. K.A.E-F. contributed to the analysis of the results. R.N., K.A.E-F., and M.A-L. contributed to the writing of the manuscript. M.E.E., M.H.E., and E.C. contributed to the writing and revising of the manuscript.

\section{Funding}

This research received no specific grant from any funding agency in the public, commercial or not-for-profit sectors.

\section{Conflict of Interest}

The authors declare that they have no conflict of interest.

\section{Data Availability}

The datasets generated and/or analysed during this study are available from the corresponding author on reasonable request.

\section{References}

1. Alshamrani M, Almalki A, Qureshi M et al. Polypharmacy and medicationrelated problems in hemodialysis patients: a call for deprescribing. Pharmacy (Basel) 2018; 6: 76. http://doi.org/10.3390/pharmacy6030076.

2. Ghimire S, Castelino RL, Lioufas NM et al. Nonadherence to medication therapy in haemodialysis patients: a systematic review. PLoS One 2015; 10: e0144119. http://doi.org/10.1371/journal.pone.0144119

3. Karkar A. Modalities of hemodialysis: quality improvement. Saudi J Kidney Dis Transpl 2012;23:1145-61. http://doi.org/10.4103/1319-2442.103553

4. Liabeuf S, Sajjad A, Kramer A et al. Guideline attainment and morbidity/ mortality rates in a large cohort of European haemodialysis patients (EURODOPPS). Nephrol Dial Transplant 2019; 34: 2105-10. http://doi. org/10.1093/ndt/gfz049

5. Chirakarnjanakorn S, Navaneethan SD, Francis GS et al. Cardiovascular impact in patients undergoing maintenance hemodialysis: clinical management considerations. Int J Cardiol 2017; 232: 12-23. http://doi. org/10.1016/j.ijcard.2017.01.015

6. Brunelli SM, Sibbel S, Colson C et al. Medicare advantage associated with lower mortality for incident dialysis patients. Nephrol News Issues 2015; 29: 16-7, 21, 26-9. 
7. Chan MW, Cheah HM, Mohd Padzil MB. Multidisciplinary education approach to optimize phosphate control among hemodialysis patients. Int J Clin Pharm 2019; 41: 1282-9. http://doi.org/10.1007/ s11096-019-00878-4

8. Porter AC, Fitzgibbon ML, Fischer MJ et al. Rationale and design of a patient-centered medical home intervention for patients with end-stage renal disease on hemodialysis. Contemp Clin Trials 2015; 42: 1-8. http:// doi.org/10.1016/j.cct.2015.02.006

9. McCann L, Hoefs M. Multidisciplinary team care for CKD-MBD. Achieving KDIGO guideline recommendations in the bundling era. Nephrol News Issues 2014; 28: 24-6, 28, 30-2 passim.

10. Pai AB, Cardone KE, Manley HJ et al.; Dialysis Advisory Group of American Society of Nephrology. Medication reconciliation and therapy management in dialysis-dependent patients: need for a systematic approach. Clin J Am Soc Nephrol 2013; 8: 1988-99. http://doi.org/10.2215/ CJN.01420213

11. Ledger S, Choma G. Medication reconciliation in hemodialysis patients. CANNT J 2008; 18: 41-3.

12. Salgado TM, Moles R, Benrimoj SI et al. Exploring the role of renal pharmacists in outpatient dialysis centres: a qualitative study. Int J Clin Pharm 2012; 34: 569-78. http://doi.org/10.1007/s11096-012-9645-z

13. St Peter WL. Management of polypharmacy in dialysis patients. Semin Dial 2015; 28: 427-32. http://doi.org/10.1111/sdi.12377

14. Mirkov S. Implementation of a pharmacist medication review clinic for haemodialysis patients. N Z Med J 2009; 122: 25-37.

15. Tang I, Vrahnos D, Hatoum $\mathrm{H}$ et al. Effectiveness of clinical pharmacist interventions in a hemodialysis unit. Clin Ther 1993; 15: 459-64; discussion 432.

16. Grabe DW, Low CL, Bailie GR et al. Evaluation of drug-related problems in an outpatient hemodialysis unit and the impact of a clinical pharmacist. Clin Nephrol 1997; 47: 117-21.

17. Ohnishi J, Miyake A, Kuwatsuka K et al. Effect of pharmacist management on serum hemoglobin levels with renal anemia in hemodialysis outpatients. Biol Pharm Bull 2011; 34: 1609-12. http://doi.org/10.1248/ bpb.34.1609

18. To LL, Stoner CP, Stolley SN et al. Effectiveness of a pharmacistimplemented anemia management protocol in an outpatient hemodialysis unit. Am J Health Syst Pharm 2001; 58: 2061-5. http://doi.org/10.1093/ ajhp/58.21.2061

19. Dashti-Khavidaki S, Khalili H, Shahverdi S et al. The role of clinical pharmacy services in achieving treatment targets in Iranian haemodialysis patients. Singapore Med J 2012; 53: 599-603.

20. Al Raiisi F, Stewart D, Fernandez-Llimos F et al. Clinical pharmacy practice in the care of Chronic Kidney Disease patients: a systematic review. Int J Clin Pharm 2019; 41: 630-66. http://doi.org/10.1007/ s11096-019-00816-4
21. Almeman A, Al-Jedai A. Pharmacy practice in the Kingdom of Saudi Arabia. In: Fathelrahman AI, Ibrahim MI, Wertheimer AI (eds.), Pharmacy Practice in Developing Countries: Achievements and Challenges. Boston: Academic Press, 2016, 171-97.

22. Elsayed TM, Elsisi GH, Elmahdawy M. Pharmacy practice in Egypt. In: Fathelrahman AI, Ibrahim MI, Wertheimer AI (eds.), Pharmacy Practice in Developing Countries: Achievements and Challenges. Boston: Academic Press, 2016, 291-317.

23. Stebbins M, Cutler T, Parker P. Assessment of therapy and medication therapy management. In: Alldredge BK, Jacobson PA, Corelli RL et al. (eds), Koda-Kimble Young's Applied Therapeutics: The Clinical Use of Drugs. Philadelphia, USA: Lippincott Williams \& Wilkins, 2013, 1-15.

24. Pharmaceutical Care Network Europe Foundation. Classification of Drug Related Problems The PCNE Classification 2017;V8.02:1-10. https:// www.pcne.org/upload/files/230_PCNE_classification_V8-02.pdf (18 May 2020, date last accessed).

25. Kidney Disease: Improving Global Outcomes (KDIGO) CKD-MBD Update Work Group. KDIGO 2017 clinical practice guideline update for the diagnosis, evaluation, prevention, and treatment of chronic kidney disease-mineral and bone disorder (CKDMBD). Kidney Int Suppl 2017; 7: 1-59.

26. Kidney Disease: Improving Global Outcomes (KDIGO) Anemia Work Group. KDIGO clinical practice guideline for anemia in chronic kidney disease. Kidney Int Suppl 2012; 2: 279-335.

27. Roberts-Clary S, Larkin JW, Matzke GR et al. Improvements in MBD lab outcomes associated with improved pharmaceutical care in hemodialysis patients. Nephrol News Issues 2017; 31: 26, 28-32.

28. Baker T, Naylor H, MacNeil B et al. Evaluation of a pharmacist-dieticianled patient-centered approach to managing CKD-MBD: a mixed-method study. Pharmacy (Basel) 2020; 8: 171.

29. Weinhandl ED, Arneson TJ, St Peter WL. Clinical outcomes associated with receipt of integrated pharmacy services by hemodialysis patients: a quality improvement report. Am J Kidney Dis 2013; 62: 557-67. http:// doi.org/10.1053/j.ajkd.2013.02.360

30. Abdel-Latif MM, Sabra K. Clinical pharmacy practice in Egyptian hospitals. Am J Health Syst Pharm 2016; 73: e63-6. http://doi.org/10.2146/ajhp150250

31. Pai AB, Boyd A, Depczynski J et al. Reduced drug use and hospitalization rates in patients undergoing hemodialysis who received pharmaceutical care: a 2-year, randomized, controlled study. Pharmacotherapy 2009; 29: 1433-40. http://doi.org/10.1592/phco.29.12.1433

32. Anderson NE, Calvert M, Cockwell $\mathrm{P}$ et al. The use of patient-reported outcomes in patients treated with maintenance hemodialysis: a perspective. Am J Kidney Dis 2019; 74: 399-406. http://doi.org/10.1053/j. ajkd.2019.01.035

33. Weisbord SD. Patient-centered dialysis care: depression, pain, and quality of life. Semin Dial 2016; 29: 158-64. http://doi.org/10.1111/sdi.12464 